\title{
Lack of impact of polycystic kidney disease on the outcome of aneurysmal subarachnoid hemorrhage: a matched case-control study
}

\author{
Heidi J. Nurmonen, BM, ${ }^{1-3}$ Terhi Huttunen, MD, PhD, ${ }^{1}$ Jukka Huttunen, MD, PhD, ${ }^{1}$ \\ Arttu Kurtelius, MD, PhD, ${ }^{1,2}$ Satu Kotikoski, BM,1,2 Antti Junkkari, MD, PhD, ${ }^{1}$ \\ Timo Koivisto, MD, PhD, ,2 Mikael von und zu Fraunberg, MD, PhD,1,2 \\ Olli-Pekka Kämäräinen, MD, PhD, ${ }^{1,2}$ Maarit Lång, MD, ,2,4 Helena Isoniemi, MD, PhD, ${ }^{6}$ \\ Juha E. Jääskeläinen, MD, PhD, ${ }^{1,2}$ and Antti E. Lindgren, MD, PhD ${ }^{1,2,5}$
}

\begin{abstract}
${ }^{1}$ Neurosurgery of NeuroCenter, Kuopio University Hospital and University of Eastern Finland, Kuopio; ${ }^{2}$ School of Medicine, Institute of Clinical Medicine, Faculty of Health Sciences, University of Eastern Finland, Kuopio; ${ }^{3}$ Kuopio Health Center, Kuopio; ${ }^{4}$ Neurointensive Care and ${ }^{5}$ Department of Clinical Radiology, Kuopio University Hospital, Kuopio; and ${ }^{6}$ Transplantation and Liver Surgery Clinic, Abdominal Center, Helsinki University Hospital, Helsinki, Finland
\end{abstract}

OBJECTIVE The authors set out to study whether autosomal dominant polycystic kidney disease (ADPKD), an established risk factor for intracranial aneurysms (IAs), affects the acute course and long-term outcome of aneurysmal subarachnoid hemorrhage (aSAH).

METHODS The outcomes of 32 ADPKD patients with aSAH between 1980 and 2015 (median age 43 years; 50\% women) were compared with 160 matched (age, sex, and year of aSAH) non-ADPKD aSAH patients in the prospectively collected Kuopio Intracranial Aneurysm Patient and Family Database.

RESULTS At 12 months, $75 \%$ of the aSAH patients with ADPKD versus $71 \%$ of the matched-control aSAH patients without ADPKD had good outcomes (Glasgow Outcome Scale score 4 or 5). There was no significant difference in condition at admission. Hypertension had been diagnosed before aSAH in $69 \%$ of the ADPKD patients versus $27 \%$ of controls $(p<0.001$ ). Multiple IAs were present in $44 \%$ of patients in the ADPKD group versus $25 \%$ in the control group ( $p$ $=0.03)$. The most common sites of ruptured IAs were the anterior communicating artery $(47 \%$ vs $29 \%, p=0.05)$ and the middle cerebral artery bifurcation ( $28 \%$ vs $31 \%)$, and the median size was $6.0 \mathrm{~mm}$ versus $8.0 \mathrm{~mm}(\mathrm{p}=0.02)$. During the median follow-up of 11 years, a second aSAH occurred in 3 of $29(10 \%)$ ADPKD patients and in 4 of $131(3 \%)$ controls $(p=0.11)$. A fatal second aSAH due to a confirmed de novo aneurysm occurred in $2(6 \%)$ of the ADPKD patients but in none of the controls $(p=0.027)$.

CONCLUSIONS The outcomes of ADPKD patients with aSAH did not differ significantly from those of matched nonADPKD aSAH patients. ADPKD patients had an increased risk of second aSAH from a de novo aneurysm, warranting long-term angiographic follow-up.

https://thejns.org/doi/abs/10.3171/2020.4.JNS20544

KEYWORDS ruptured intracranial aneurysm; subarachnoid hemorrhage; polycystic kidney; autosomal dominant; vascular disorders

A UTOSOMAL dominant polycystic kidney disease (ADPKD), characterized by bilateral enlarged cystic kidneys, is a serious multisystem disease. ${ }^{1}$ In most cases caused by a PKD1 or PKD2 mutation, it is carried by 1:1000-1:2500 according to population sequencing. ${ }^{2,3}$ In $10 \%-25 \%$ of patients, there is no clear family history. ${ }^{4}$ Clinical penetration and the course of renal disease and other manifestations vary, even within an ADPKD family. ${ }^{5}$ The majority of ADPKD patients develop kidney failure requiring dialysis at a median age of 55 years ${ }^{6,7}$ often followed by kidney transplantation. A vasopressin V2 antagonist may delay disease progression. ${ }^{8}$ Hypertension is present already in $35 \%$ of patients before the age of 18 years, and in $60 \%$ it precedes a decline in renal function. ${ }^{9}$

ABBREVIATIONS AComA = anterior communicating artery; ADPKD = autosomal dominant polycystic kidney disease; aSAH = aneurysmal subarachnoid hemorrhage; GOS = Glasgow Outcome Scale; IA = intracranial aneurysm; KUH = Kuopio University Hospital; MCA = middle cerebral artery.

SUBMITTED February 28, 2020. ACCEPTED April 15, 2020.

INCLUDE WHEN CITING Published online July 3, 2020; DOI: 10.3171/2020.4.JNS20544. 
ADPKD predisposes patients to several cardiovascular manifestations, e.g., valve insufficiency, left ventricular hypertrophy, and aneurysms, and dissections in extracranial and intracranial arteries. ${ }^{5}$

Aneurysmal subarachnoid hemorrhage (aSAH), usually from rupture of a saccular intracranial aneurysm (IA), has an incidence of 9 per 100,000 patient-years ${ }^{10}$ and a peak age in the 6th decade of life. ${ }^{11}$ Risk factors include smoking, hypertension, female sex, family history of saccular IA, and ADPKD. ${ }^{11}$

As has been established, approximately $10 \%$ of ADPKD patients harbor unruptured IAs in their lifetime. ${ }^{12}$ Up to $6 \%$ of ADPKD patients might die of aSAH..$^{13}$ In a recent prospective series of 495 ADPKD patients, the incidence of SAH was 2.0 per 1000 patient-years. ${ }^{14}$ Family history of IA disease ${ }^{12}$ and a mutation near the $5^{\prime}$ end of $P K D I^{15}$ are the only known risk factors for IAs within this population, and their absence does not rule out the possibility of IAs. ADPKD patients experience aSAH approximately 10 years earlier than the general population, and their aneurysms are smaller at rupture. ${ }^{16}$ These patients also seem to be at an increased risk of developing de novo aneurysms after surviving aSAH. ${ }^{16}$

The Kuopio Intracranial Aneurysm Patient and Family Database contains data on all patients with ruptured and unruptured IAs who were admitted to the Kuopio University Hospital (KUH) from a defined Eastern Finnish population since 1980. Here, we compared 32 ADPKD patients with 160 matched (age at aSAH, year of aSAH, and sex) non-ADPKD patients with their first aSAH. Our aim was to evaluate the impact of ADPKD on the acute course and long-term outcome of aSAH.

\section{Methods}

\section{Catchment Population of KUH}

Finland is divided into catchment areas of 5 publicly funded university hospitals. Kuopio Neurosurgery and Neurointensive Care have solely provided acute and elective neurosurgical care for the Eastern Finnish catchment population. Between 1980 and 2015, the population decreased from 824,823 to 815,093 while the geographic area remained unchanged; the proportion of males increased from $49 \%$ to $49.5 \% .^{16}$

\section{Management Protocol of aSAH in Eastern Finland}

All SAH patients, diagnosed by CT scanning or lumbar puncture, are transferred acutely to KUH for neurointensive care, 4-vessel digital subtraction angiography, and neurosurgical care, including microsurgical or endovascular IA occlusion if not moribund or very aged. Neurointensive care is provided regardless of the condition on admission, including comatose Hunt and Hess grade $\mathrm{V}$ patients, by a team of neurointensivists, neurosurgeons, and neuroradiologists according to international recommendations. ${ }^{17}$

\section{The Kuopio Intracranial Aneurysm Patient and Family Database}

KUH Neurosurgery maintains a database, prospective since 1990, of all cases of patients with angiographically verified ruptured or unruptured IAs, admitted to KUH since 1980 . The database is managed by a full-time coordinator, who interviews all patients and codes the data, including family history of IA, hospital periods, and followup visits. Clinical data, including the hospital diagnoses, prescribed medicines, and causes of death, are obtained from the national registries using unique social security numbers since 1995. The database, including 5250 IA patients at present, has been extensively studied for phenotype on admission, risk factors, concomitant diseases, outcome, and IA genetics. ${ }^{18-23}$

\section{Identification of IA Patients With ADPKD}

Between 1980 and 2015, 4436 patients with IA were admitted to KUH Neurosurgery from the Eastern Finnish catchment population. In the KUH IA database, 53 IA patients with ADPKD were identified and consisted of those who 1) had been diagnosed with ADPKD before IA; 2) had ADPKD confirmed at autopsy after aSAH; 3) were diagnosed with ADPKD after IA and informed the nurse coordinator of the database of this at a control visit; and 4) have had an ICD-10 diagnosis code of either Q61.2 (polycystic kidney, adult type) or Q61.3 (polycystic kidney, nonspecified) in the Finnish national database of hospital diagnoses. Their patient records were carefully reviewed. ${ }^{16}$ Thirty-three patients presented with aSAH and 20 with an unruptured IA. One patient was excluded, giving us the final study cohort of 32 ADPKD patients with aSAH.

\section{Current Study Groups}

The study group includes the ADPKD patients admitted alive to KUH between 1980 and 2015 with their first aSAH. The control group includes 5 non-ADPKD control patients admitted alive to KUH between 1980 and 2015 with their first aSAH for each ADPKD aSAH patient, matched by sex, age at aSAH, and year of aSAH (Fig. 1). In case-control matching (IBM SPSS Statistics version 22.0, IBM Corp.), priority was given to exact matches, but a range of 3 years in age and 5 years for the year of aSAH were allowed for a sufficient number of controls. The records of the controls were rereviewed to exclude ADPKD and family history of ADPKD.

\section{Variables}

The variables used to compare the groups are presented in Tables 1-3. Familial saccular IA disease was defined as $\geq 2$ affected first-degree relatives. Hypertension was defined as one of the following: diagnosis of hypertension, antihypertensive medication started before aSAH, or self-reported diagnosis. Kidney function was defined by the glomerular filtration rate, estimated using the CKDEPI (Chronic Kidney Disease-Epidemiology Collaboration) formula. ${ }^{24}$ Data on dialysis or kidney transplantation, before or after aSAH, were obtained from the medical records and national clinical data, including the Finnish National Transplantation Registry.

\section{Statistical Analysis}

Categorical variables are expressed as proportions and continuous variables as medians, IQRs, and ranges. The 2 


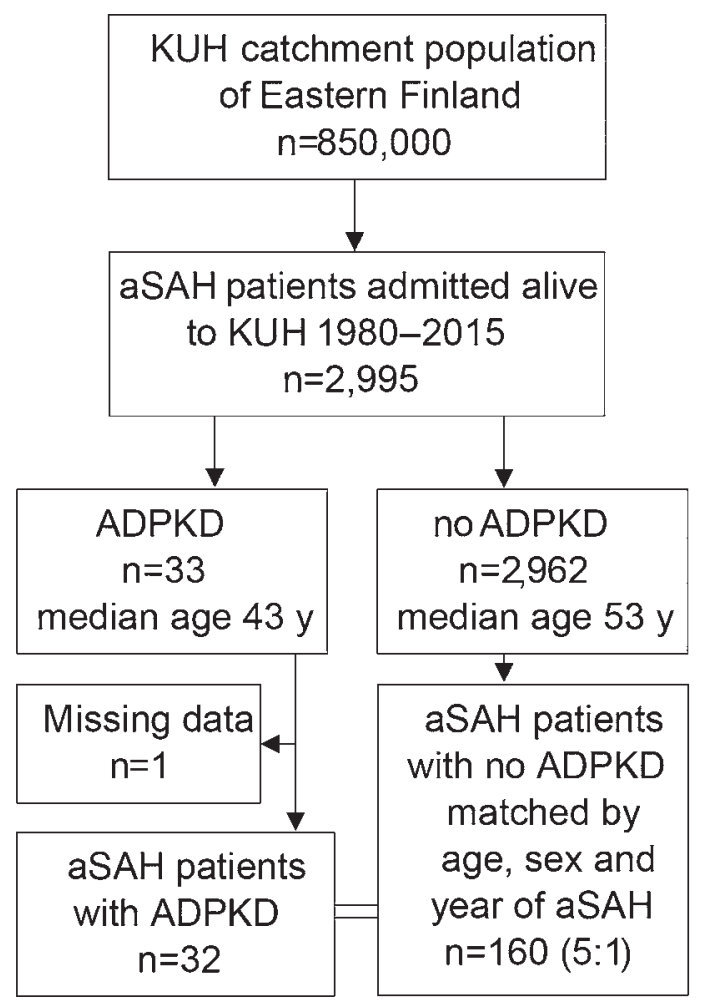

FIG. 1. Flowchart of patient inclusion in the present study.

groups were compared using the chi-square test, Fisher's exact test, Mood's median test, and Mann-Whitney U-test, as appropriate; $\mathrm{p} \leq 0.05$ was considered significant. Multivariate analysis was not performed due to the small num- ber of ADPKD patients. Kaplan-Meier analysis with the log-rank test was used to compare the cumulative mortality rates after aSAH. In survival analysis, follow-up was calculated from the date of SAH until death or the last date of follow-up. IBM SPSS Statistics (version 22.0, IBM Corp.) was used for statistical analyses.

\section{Ethical Issues}

This study was approved by the ethics committee of KUH. The study was conducted according to the Declaration of Helsinki, and all patients provided informed consent. Data fusion from the Finnish nationwide registries was approved by the Ministry of Social Affairs and Health of Finland.

\section{Results \\ Patient Groups}

There were 32 ADPKD patients with aSAH, 16 females and 16 males (1:1), with median ages of 44 years and 39 years, respectively (Fig. 1 and Table 1). The patients were compared with the 160 matched non-ADPKD control aSAH patients. Hypertension was diagnosed before aSAH in $69 \%(22 / 32)$ of the ADPKD patients and in $27 \%$ $(43 / 160)$ of the controls ( $\mathrm{p}<0.001)$. Among the 32 ADPKD patients, $19 \%(6 / 32)$ had moderately to severely decreased kidney function at aSAH, none were on dialysis, and 1 patient already had received a donor kidney. Twenty-seven percent of the ADPKD patients had normal kidney function at the time of admission for aSAH, and in $56 \%$, the serum creatinine level was within the normal range.

\section{Clinical Condition on Admission}

The ruptured aneurysms of ADPKD patients were sig-

TABLE 1. Baseline characteristics of the 32 study patients and the 160 matched control patients

\begin{tabular}{|c|c|c|c|}
\hline Variable & $\begin{array}{l}\text { aSAH Patients w/ } \\
\text { ADPKD }(n=32)\end{array}$ & $\begin{array}{c}\text { Matched aSAH } \\
\text { Patients w/o ADPKD } \\
(n=160 ; 5: 1)\end{array}$ & p Value \\
\hline Female sex, n (\%) & $16(50)$ & $80(50)$ & Matched \\
\hline Median age on admission, yrs (IQR) & $43(34-51)$ & $43(34-51)$ & Matched \\
\hline Familial sIA disease, $\mathrm{n}(\%)$ & $0(0)$ & $18(11)$ & 0.047 \\
\hline Smoking, current or former, n (\%) & $10(31)$ & $63(39)$ & NS \\
\hline Hypertension diagnosed before aSAH, n (\%) & $22(69)$ & $43(27)$ & $<0.001$ \\
\hline Diabetes diagnosed before aSAH, n (\%) & $0(0)$ & $8(5)$ & NS \\
\hline Median age at ADPKD diagnosis, yrs (IQR)* & $42(30-53)$ & & \\
\hline \multicolumn{4}{|l|}{ Kidney function on admission† } \\
\hline Median eGFR, ml/min (IQR) & $83(61-98)$ & $98(85-108)$ & 0.005 \\
\hline Normal function ( $\geq 90), \mathrm{n}(\%)$ & $7(27)$ & $86(67)$ & $<0.001$ \\
\hline Mild decrease (60-89), n (\%) & $13(50)$ & $39(30)$ & \\
\hline Moderate decrease (30-59), n (\%) & $5(19)$ & $3(2)$ & \\
\hline Severe decrease (15-29), n (\%) & $1(4)$ & $0(0)$ & \\
\hline Kidney failure (<15), n (\%) & $0(0)$ & $0(0)$ & \\
\hline Kidney transplantation/dialysis before aSAH, n & $1 / 0$ & $0 / 0$ & \\
\hline
\end{tabular}


TABLE 2. Clinical condition on admission to neurointensive care of 32 ADPKD patients and 160 matched control patients with aSAH

\begin{tabular}{|c|c|c|c|}
\hline Variable & $\begin{array}{l}\text { aSAH Patients w/ } \\
\text { ADPKD }(n=32)\end{array}$ & $\begin{array}{l}\text { Matched aSAH Patients } \\
\text { w/o ADPKD }(n=160)\end{array}$ & $\mathrm{p}$ Value \\
\hline \multicolumn{4}{|l|}{ Hunt \& Hess grade, $n(\%)$} \\
\hline I & $4(12)$ & $17(11)$ & NS \\
\hline II & $13(41)$ & $72(45)$ & NS \\
\hline III & $8(25)$ & $29(18)$ & NS \\
\hline IV & $6(19)$ & $31(19)$ & NS \\
\hline V & $1(3)$ & $10(6)$ & NS \\
\hline \multicolumn{4}{|l|}{ Acute CT findings, $\mathrm{n}(\%)$} \\
\hline Subdural hematoma & $2(6)$ & $7(4)$ & NS \\
\hline Intracerebral hemorrhage & $6(19)$ & $45(28)$ & NS \\
\hline Intraventricular hemorrhage & $8(25)$ & $53(34)$ & NS \\
\hline Hydrocephalus & $13(41)$ & $72(46)$ & NS \\
\hline Extraventricular drainage, $\mathrm{n}(\%)$ & $11(34)$ & $51(32)$ & NS \\
\hline Meningitis treated, $\mathrm{n}(\%)$ & $1(3)$ & $21(13)$ & NS \\
\hline \multicolumn{4}{|l|}{ Delayed cerebral ischemia, $\mathrm{n}(\%)$} \\
\hline Vasospasm on angiography & $5(16)$ & $37(23)$ & NS \\
\hline Delayed cerebral ischemia clinically & $7(22)$ & $38(24)$ & NS \\
\hline Both of the above & $4(12)$ & $25(16)$ & NS \\
\hline \multicolumn{4}{|l|}{ Site of ruptured IA, n (\%) } \\
\hline AComA & $15(47)$ & $47(29)$ & 0.054 \\
\hline$A_{2}-A_{5}$ & $1(3)$ & $7(4)$ & NS \\
\hline MCA bifurcation & $9(28)$ & $49(31)$ & NS \\
\hline Other MCA segments & $3(9)$ & $10(6)$ & NS \\
\hline ICA & $3(9)^{*}$ & $12(8)$ & NS \\
\hline PComA & $0(0)$ & $17(11)$ & 0.054 \\
\hline BA bifurcation & $0(0)$ & $5(3)$ & NS \\
\hline Other & $1(3)$ & $13(8) \dagger$ & NS \\
\hline \multicolumn{4}{|l|}{ Size of ruptured IA, mm‡ } \\
\hline Median & 6.0 & 8.0 & 0.022 \\
\hline$<5, \mathrm{n}(\%)$ & $10(32)$ & $24(16)$ & 0.032 \\
\hline $5-10, n(\%)$ & $17(55)$ & $84(55)$ & NS \\
\hline$>10, \mathrm{n}(\%)$ & $4(13)$ & $44(29)$ & NS \\
\hline Multiple IAs, n (\%) & $14(44)$ & $40(25)$ & 0.031 \\
\hline Ruptured IA etiology, n (\%) & & & NS \\
\hline Saccular & 31 & 158 & \\
\hline Fusiform & 1 & 2 & \\
\hline Wall, $n(\%)$ & & & NS \\
\hline Irregular & $29(91)$ & $146(91)$ & \\
\hline Smooth & $2(6)$ & $11(7)$ & \\
\hline Not available & $1(3)$ & $3(2)$ & \\
\hline \multicolumn{4}{|l|}{ Treatment of ruptured IA, $n(\%)$} \\
\hline Open microsurgery & $25(78)$ & $120(75)$ & NS \\
\hline Endovascular & $4(12)$ & $29(18)$ & NS \\
\hline Conservative & $3(9)$ & $11(7)$ & NS \\
\hline
\end{tabular}

$\mathrm{BA}=$ basilar artery $; \mathrm{ICA}=$ internal carotid artery; $\mathrm{PComA}=$ posterior communicating artery.

* One IA was fusiform.

$\dagger$ Two IAs were fusiform.

$\ddagger$ Size was not known in 1 patient in the ADPKD group and 8 in the non-ADPKD group. 
TABLE 3. Outcome of 32 ADPKD patients and 160 matched control patients after aSAH

\begin{tabular}{|c|c|c|c|}
\hline Variable & $\begin{array}{l}\text { aSAH Patients w/ } \\
\text { ADPKD }(n=32)\end{array}$ & $\begin{array}{l}\text { Matched aSAH Patients } \\
\text { w/o ADPKD }(n=160)\end{array}$ & p Value \\
\hline \multicolumn{4}{|l|}{ Mortality, n (\%) } \\
\hline at $1 \mathrm{mo}$ & $3(9)$ & $22(14)$ & NS \\
\hline at $12 \mathrm{mos}$ & $3(9)$ & $29(18)$ & NS \\
\hline at $5 \mathrm{yrs}$ & $5(16)$ & $34(21)$ & NS \\
\hline at $10 \mathrm{yrs}$ & $8(25)$ & $44(28)$ & NS \\
\hline \multicolumn{4}{|l|}{ Leading causes of death, $n(\%)$} \\
\hline aSAH w/ late effects & $6(19)$ & $32(20)$ & \\
\hline Cancer & & $10(6)$ & \\
\hline ADPKD & $3(9)$ & & \\
\hline Cardiovascular disease & $2(6)$ & $8(5)$ & \\
\hline Good outcome (GOS 4 or 5) at 12 mos, $n(\%)$ & $24(75)$ & $114(71)$ & NS \\
\hline Poor outcome (GOS 1-3) at 12 mos, n (\%) & $8(25)$ & $46(29)$ & NS \\
\hline Second SAH after surviving 12 mos, $n(\%)$ & $3 / 29(9)$ & $4 / 131(3)$ & 0.092 \\
\hline Returned to previous profession, $n(\%)^{*}$ & $18(60)$ & $78(54)$ & NS \\
\hline Median age at death, yrs (range) & $64(33-82)$ & $58(20-92)$ & NS \\
\hline
\end{tabular}

nificantly smaller than those of the control patients (median 6.0 vs $8.0 \mathrm{~mm}, \mathrm{p}=0.022$ ) (Table 2). There were more small $(<5 \mathrm{~mm})$ ruptured IAs in the ADPKD patients than in the control group (32\% vs $16 \%, \mathrm{p}=0.032)$. Presenting with multiple aneurysms at initial diagnosis was more common among the ADPKD patients $(44 \%$ vs $25 \%$, $\mathrm{p}=$ $0.031)$. There were no statistically significant differences in aneurysm location or aneurysm shape between the groups. Distribution of saccular and fusiform aneurysms was similar.

\section{Aneurysmal SAH Presentation}

The median Hunt and Hess grade at admission among both groups was II: $41 \%$ among the ADPKD patients and $45 \%$ among the controls. We found no statistically significant differences in the prevalence of aSAH complications, such as intracerebral hematoma or delayed ischemic complication. Clinical characteristics of aSAH in the 2 groups are presented in Table 2.

\section{Outcome at 12 Months}

At 12 months, 9\% in the ADPKD group versus 18\% in the non-ADPKD group had died $(\mathrm{p}=0.23)$, while $75 \%$ versus $71 \%$ had good functional outcome (Glasgow Outcome Scale [GOS] score 4 or 5) (Table 3). Outcome was poor (GOS score $1-3)$ in $25 \%$ versus $29 \%(\mathrm{p}=0.67) ; 60 \%$ versus $54 \%$ of the individuals working before aSAH returned to their previous occupation.

\section{Recurrent Hemorrhage}

In total, a second SAH occurred in $3 \operatorname{ADPKD}(2,7$, and 10 years after the initial hemorrhage) and 4 control $(2,6$, 10 , and 22 years) patients $(p=0.06)$, which was in 2 ADPKD patients (patients $A$ and $B$ ) but in none of the control patients from a confirmed de novo aneurysm $(\mathrm{p}=0.03)$. The recurrent SAH was fatal in 5 of the 7 (71\%) patients.
Patient A was a female with normal kidney function, a smoker, with no hypertension, who experienced aSAH at the age of 22 years due to an 8 -mm aneurysm in the middle cerebral artery (MCA). Ten years later, the patient had a second aSAH due to a 5-mm de novo aneurysm of the anterior communicating artery (AComA). She also had 2 new unruptured de novo IAs (basilar artery and internal carotid artery). She survives and works in her previous profession. Patient B was a female with normal kidney function, a smoker with hypertension who had aSAH at the age of 26 years due to an MCA aneurysm. She recovered well apart from epilepsy and returned to her previous profession. Seven years years later, now with end-stage kidney failure, she had a second aSAH due to a 4-mm de novo aneurysm of the pericallosal artery, and also an unruptured de novo IA (AComA). The second aSAH was fatal.

\section{Outcome After 12 Months}

The median follow-up time was 15 years (447 patientyears) for the 29 ADPKD patients and 17 years (2266 patient-years) for the 131 non-ADPKD patients alive at 12 months (Table 3). At 10 years, the cumulative survival rates were $80 \%$ versus $85 \%$ (Fig. 2). Shunt-dependent hydrocephalus developed in $6 \%$ versus $11 \%$ of patients. Nine (31\%) ADPKD patients had a kidney transplant at a median of 10 years after aSAH (range 3-23 years).

\section{Discussion}

In this matched case-control study, we found no difference in aSAH outcome between ADPKD aSAH patients and their matched controls. This is the first study to compare the phenotype and outcome of aSAH between ADPKD patients and a 5-fold matched population control group of aSAH patients without ADPKD.

In our study, case fatality in ADPKD patients with first aSAH was $9 \%$ at 12 months. In previous cohorts, 
case fatalities ranging from $10 \%$ at 3 months ${ }^{25}$ to $55 \%$ at 6 months ${ }^{26}$ have been reported. In a meta-analysis of 160 aSAH patients, $43 \%$ were dead and $21 \%$ were dependent. ${ }^{27}$ Aneurysmal SAH is diverse in presentation, and the best predictor of outcome is the level of consciousness on admission. ${ }^{28,29}$ In our cohort of young (median age 43 years) aSAH patients, the median Hunt and Hess grade was II in both groups, and only $22 \%$ of the ADPKD patients versus $26 \%$ of the controls had a Hunt and Hess grade of IV or V. In this study, ADPKD patients had aSAH from smaller aneurysms than matched controls $(6.0 \mathrm{~mm}$ vs $8.0 \mathrm{~mm})$. This is in line with previous data. ${ }^{16,25,26}$

In our study, ADPKD patients fared similarly to their matched controls. Previously, occurrences of preexisting medical conditions worsening aSAH prognosis have been reported, such as coronary artery disease and chronic obstructive airway disease ${ }^{30}$ Chronic kidney disease is associated with a higher incidence of stroke and is a risk factor for poor outcome in both ischemic and hemorrhagic stroke, ${ }^{31}$ however, data on SAH as a separate entity from hemorrhagic stroke, including intracerebral hemorrhage, are sparse. According to an analysis on 1.1 million patients from the Nationwide Inpatient Sample, the adjusted OR for in-hospital death when hospitalized with SAH was 1.93 for chronic kidney disease patients. ${ }^{32}$ None of our ADPKD patients was in terminal kidney failure at aSAH, which is likely related to their younger age at $\mathrm{aSAH}^{16}$ than that at which they reach end-stage renal failure. ${ }^{6,7}$ One ADPKD patient experienced aSAH despite having already received a donor kidney, but even so their outcome was excellent. These results indicate that kidney failure is usually not a major factor in ADPKD patients with SAH, and other factors are more important in determining the outcome. Surprisingly, life expectancy after aSAH was not different between ADPKD patients and matched controls. This is the first study on this subject.

In this study, ADPKD patients were at an increased risk of having a second aSAH from a de novo aneurysm. In a large international cohort of European ADPKD aSAH patients, 3 of 47 (6.4\%) experienced a second aSAH from a de novo aneurysm during a mean follow-up of 8.3 years..$^{25}$ This is also in line with our previous finding that the rate of de novo aneurysm formation is $1.3 \%$ per patient-year among ADPKD patients after surviving aSAH and $0.2 \%$ per patient-year in the general Eastern Finnish aSAH population (HR 7.7). ${ }^{16}$ A recent systematic review suggested that the de novo formation rate in ADPKD patients is as high as $1.4 \%$ per patient-year. ${ }^{33}$ Our results again highlight the importance of angiographic follow-up in this subgroup of aSAH patients.

This study has several strengths derived from the universal and publicly funded healthcare system in Finland. The country is divided into mutually exclusive catchment populations between the 5 university hospitals, allowing unselected and minimally biased disease databases. The Kuopio Intracranial Aneurysm Patient and Family Database, with clinical data integrated from the national registries, using the personal identification codes, allows for retrospective construction of complete clinical lifelines of aSAH patients from Eastern Finland from birth to death or last follow-up. ${ }^{34-38}$

This is a retrospective study, and as such is subject to se-

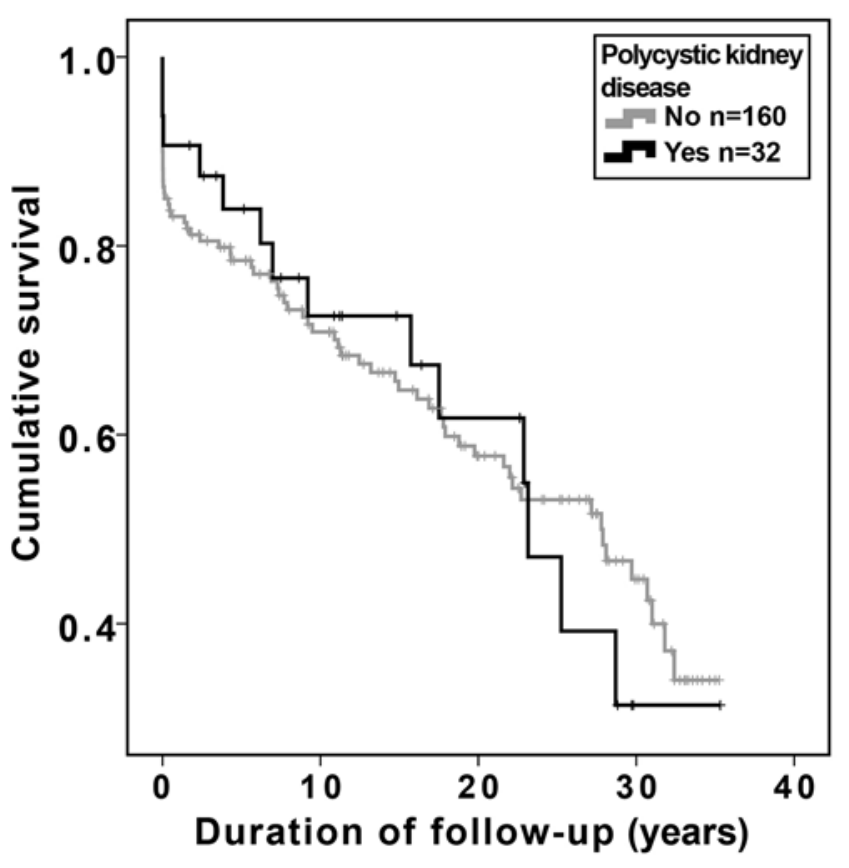

FIG. 2. Kaplan-Meier survival curve after aSAH in 32 ADPKD patients and 160 matched controls.

lection bias. However, the unselected database from which the study population is derived, along with the 1:5 matched control group, compensates for this. Furthermore, to minimize the possibility of incorrect diagnoses of ADPKD, the files of the 192 patients were rereviewed.

ADPKD is a rare disease and subsequently the study population is relatively small, which could have contributed to our negative finding. However, we feel that this is unlikely, given the fact that control patients in fact had a slightly higher mortality and percentage of poor outcome. To reach 5 matched control patients for each ADPKD patient (median age 43 years), a range of 5 years in the year of admission and a range of 3 years in age at aSAH had to be allowed, which may cause bias if the treatment of aSAH had significantly improved within that time range. There was no statistically significant difference in the proportion of endovascular versus microsurgical aneurysm occlusion. Even if there was a bias due to this, this is unlikely to be systematic, especially when priority was given to exact matches in the case-control matching.

It is possible that some aSAH patients with ADPKD have been missed, if they died before reaching the hospital or had not been admitted to KUH due to poor condition or presumed poor prognosis due to ADPKD.

In Finland, all kidney transplant candidates are screened for cerebral aneurysms before the operation; it is possible that the patients who reach uremia at a young age have had their aneurysms prophylactically treated. It should be noted that our results may not be directly applicable to other populations. Also, the impact of kidney failure on aSAH outcome should be addressed in larger cohorts.

\section{Conclusions}

There is no significant difference in aSAH prognosis in 
patients with ADPKD in comparison with matched population controls. The risk of recurrent aSAH from a de novo aneurysm is increased in ADPKD patients, indicating the need for long-term angiographic follow-up.

\section{Acknowledgments}

Virve Kärkkäinen, $\mathrm{PhD}$, facilitated the study.

This study was funded by The Finnish Kidney and Liver Association, The Petri Honkanen Foundation, The Päivikki and Sakari Sohlberg Foundation, The Maire Taponen Foundation, The North Savo Regional Fund of Finnish Cultural Foundation, Kuopio University Hospital, and The Academy of Finland.

\section{References}

1. Cornec-Le Gall E, Alam A, Perrone RD. Autosomal dominant polycystic kidney disease. Lancet. 2019;393(10174):919_ 935.

2. Willey CJ, Blais JD, Hall AK, et al. Prevalence of autosomal dominant polycystic kidney disease in the European Union. Nephrol Dial Transplant. 2017;32(8):1356-1363.

3. Lanktree MB, Haghighi A, Guiard E, et al. Prevalence estimates of polycystic kidney and liver disease by population sequencing. J Am Soc Nephrol. 2018;29(10):2593-2600.

4. Iliuta I-A, Kalatharan V, Wang K, et al. Polycystic kidney disease without an apparent family history. J Am Soc Nephrol. 2017;28(9):2768-2776.

5. Torres VE, Harris PC, Pirson Y. Autosomal dominant polycystic kidney disease. Lancet. 2007;369(9569):1287-1301.

6. Shaw C, Simms RJ, Pitcher D, Sandford R. Epidemiology of patients in England and Wales with autosomal dominant polycystic kidney disease and end-stage renal failure. Nephrol Dial Transplant. 2014;29(10):1910-1918.

7. Reule S, Sexton DJ, Solid CA, et al. ESRD from autosomal dominant polycystic kidney disease in the United States, 2001-2010. Am J Kidney Dis. 2014;64(4):592-599.

8. Blair HA. Tolvaptan: a review in autosomal dominant polycystic kidney disease. Drugs. 2019;79(3):303-313.

9. Ecder T, Schrier RW. Hypertension in autosomal-dominant polycystic kidney disease: early occurrence and unique aspects. J Am Soc Nephrol. 2001;12(1):194-200.

10. Rinkel GJE, Algra A. Long-term outcomes of patients with aneurysmal subarachnoid haemorrhage. Lancet Neurol. 2011;10(4):349-356.

11. van Gijn J, Kerr RS, Rinkel GJ. Subarachnoid haemorrhage. Lancet. 2007;369(9558):306-318.

12. Zhou Z, Xu Y, Delcourt C, et al. Is regular screening for intracranial aneurysm necessary in patients with autosomal dominant polycystic kidney disease? A systematic review and meta-analysis. Cerebrovasc Dis. 2017;44(1-2):75-82.

13. Fick GM, Johnson AM, Hammond WS, Gabow PA. Causes of death in autosomal dominant polycystic kidney disease. $J$ Am Soc Nephrol. 1995;5(12):2048-2056.

14. Flahault A, Trystram D, Nataf F, et al. Screening for intracranial aneurysms in autosomal dominant polycystic kidney disease is cost-effective. Kidney Int. 2018;93(3):716-726.

15. Rossetti S, Chauveau D, Kubly V, et al. Association of mutation position in polycystic kidney disease 1 (PKD1) gene and development of a vascular phenotype. Lancet. 2003;361(9376):2196-2201.

16. Nurmonen HJ, Huttunen T, Huttunen J, et al. Polycystic kidney disease among 4,436 intracranial aneurysm patients from a defined population. Neurology. 2017;89(18):1852-1859.

17. Kämäräinen OP, Huttunen J, Lindgren A, et al. Identification of potential organ donors after aneurysmal subarachnoid hemorrhage in a population-based neurointensive care in Eastern Finland. Acta Neurochir (Wien). 2018;160(8):15071514.
18. Lindgren AE, Räisänen S, Björkman J, et al. De novo aneurysm formation in carriers of saccular intracranial aneurysm disease in Eastern Finland. Stroke. 2016;47(5):1213-1218.

19. Lindgren AE, Kurki MI, Riihinen A, et al. Hypertension predisposes to the formation of saccular intracranial aneurysms in 467 unruptured and 1053 ruptured patients in Eastern Finland. Ann Med. 2014;46(3):169-176.

20. Huttunen J, Lindgren A, Kurki MI, et al. Antidepressant use after aneurysmal subarachnoid hemorrhage: a populationbased case-control study. Stroke. 2016;47(9):2242-2248.

21. Kotikoski S, Huttunen J, Huttunen TJ, et al. Secondary hypertension in patients with saccular intracranial aneurysm disease: a population based study. PLoS One. 2018;13(10):e0206432.

22. Adams H, Ban VS, Leinonen V, et al. Risk of shunting after aneurysmal subarachnoid hemorrhage: a collaborative study and initiation of a consortium. Stroke. 2016;47(10):24882496.

23. Kurtelius A, Kurki MI, von Und Zu Fraunberg M, et al. Saccular intracranial aneurysms in children when both parents are sporadic or familial carriers of saccular intracranial aneurysms. Neuroepidemiology. 2019;52(1-2):47-54.

24. Levey AS, Stevens LA, Schmid CH, et al. A new equation to estimate glomerular filtration rate. Ann Intern Med. 2009;150(9):604-612.

25. Chauveau D, Pirson Y, Verellen-Dumoulin C, et al. Intracranial aneurysms in autosomal dominant polycystic kidney disease. Kidney Int. 1994;45(4):1140-1146.

26. Schievink WI, Torres VE, Piepgras DG, Wiebers DO. Saccular intracranial aneurysms in autosomal dominant polycystic kidney disease. J Am Soc Nephrol. 1992;3(1):88-95.

27. Gieteling EW, Rinkel GJ. Characteristics of intracranial aneurysms and subarachnoid haemorrhage in patients with polycystic kidney disease. J Neurol. 2003;250(4):418-423.

28. Karamanakos PN, von Und Zu Fraunberg M, Bendel S, et al. Risk factors for three phases of 12-month mortality in 1657 patients from a defined population after acute aneurysmal subarachnoid hemorrhage. World Neurosurg. 2012;78(6):631639.

29. Steiner T, Juvela S, Unterberg A, et al. European Stroke Organization guidelines for the management of intracranial aneurysms and subarachnoid haemorrhage. Cerebrovasc Dis. 2013;35(2):93-112.

30. Langham J, Reeves BC, Lindsay KW, et al. Variation in outcome after subarachnoid hemorrhage: a study of neurosurgical units in UK and Ireland. Stroke. 2009;40(1):111-118.

31. Toyoda K, Ninomiya T. Stroke and cerebrovascular diseases in patients with chronic kidney disease. Lancet Neurol. 2014;13(8):823-833.

32. Ovbiagele B. Chronic kidney disease and risk of death during hospitalization for stroke. J Neurol Sci. 2011;301(1-2):46-50.

33. Cagnazzo F, Gambacciani C, Morganti R, Perrini P. Intracranial aneurysms in patients with autosomal dominant polycystic kidney disease: prevalence, risk of rupture, and management. A systematic review. Acta Neurochir (Wien). 2017;159(5):811-821.

34. Björkman J, Frösen J, Tähtinen O, et al. Aneurysm size is the strongest risk factor for intracranial aneurysm growth in the Eastern Finnish population. Neurosurgery. 2019;84(5):10981103.

35. Huttunen J, Kurki MI, von Und Zu Fraunberg M, et al. Epilepsy after aneurysmal subarachnoid hemorrhage: a population-based, long-term follow-up study. Neurology. 2015;84(22):2229-2237.

36. Huttunen J, Lindgren A, Kurki MI, et al. Epilepsy-associated long-term mortality after aneurysmal subarachnoid hemorrhage. Neurology. 2017;89(3):263-268.

37. Huttunen T, Riihinen A, Pukkala E, et al. Increased relative risk of lung cancer in 2,904 patients with saccular intracrani- 
al aneurysm disease in Eastern Finland. Neuroepidemiology. 2012;38(2):93-99.

38. Paavola JT, Väntti N, Junkkari A, et al. Antipsychotic use among 1144 patients after aneurysmal subarachnoid hemorrhage: a population-based case-control study. Stroke. 2019;50(7):1711-1718.

\section{Disclosures}

The authors report no conflict of interest concerning the materials or methods used in this study or the findings specified in this paper.

\section{Author Contributions}

Conception and design: Nurmonen, Jääskeläinen, Lindgren. Acquisition of data: Nurmonen, Isoniemi. Analysis and interpretation of data: Nurmonen. Drafting the article: Nurmonen.
Critically revising the article: Nurmonen, T Huttunen, J Huttunen, Kurtelius, Kotikoski, Junkkari, Koivisto, von und zu Fraunberg, Kämäräinen, Lång, Isoniemi, Lindgren. Reviewed submitted version of manuscript: all authors. Statistical analysis: Nurmonen. Study supervision: von und zu Fraunberg, Jääskeläinen, Lindgren.

\section{Supplemental Information}

Previous Presentations

A portion of the results was presented as an oral presentation at the 15th International Conference on Subarachnoid Hemorrhage, Amsterdam, the Netherlands, June 25-28, 2019.

\section{Correspondence}

Heidi J. Nurmonen: Kuopio University Hospital and University of Eastern Finland, Kuopio, Finland. heidi.nurmonen@kuh.fi. 